\title{
Propuesta de Medición Del DESEMPEÑO POLICIAL EN DEMOCRACIA*
}

\author{
María Eugenia Bonilla Ovallos**
}

\section{Resumen}

Tras abordar la relación existente entre policía y régimen político, y describir las características fundamentales del modelo de policía que requiere la democracia, este artículo pretende plantear un modelo de medición del desempeño policial democrático que permita a los países monitorear los avances o retrocesos que al respecto presentan los cuerpos policiales. Para ello propone el Índice de Desempeño Policial Democrático, constituido por tres dimensiones: efectividad, legitimidad y legalidad policial.

Palabras clave: policía, democracia, desempeño policial, efectividad, legalidad, legitimidad.

\section{AN INDEX OF DEMOCRATIC POLICY PERFORMANCE}

\begin{abstract}
This article discusses an Index of Democratic Policy Performance. The model builds on a conceptual discussion about the relationship between police and political regime, focusing on core police features expected in democratic settings. Index dimensions include police effectiveness, police legitimacy and policy law.
\end{abstract}

Key words: Police, democracy, institutional performance, effectiveness, legality, legitimacy.

\footnotetext{
* Este artículo es resultado de una investigación sobre desempeńo policial en El Salvador que adelanta la autora para optar al título de Doctora de la Universidad de Salamanca, España. Así mismo, cuenta con el respaldo del Instituto de Estudios Políticos de la Universidad Autónoma de Bucaramanga (UNAB), Colombia, donde se encuentra vinculado al Grupo de Investigación de Ciencia Política en las líneas de Políticas Públicas.

** Economista y especialista en Administración de Empresas, UsTA (Colombia); doctoranda en Estado de Derecho y Buen Gobierno de la usal (España). Directora del Instituto de Estudios Políticos (IEP), Universidad Autónoma de Bucaramanga (UNAB), Bucaramanga (Colombia). [mbonilla566@unab.edu.co].

Recibido: 28 de agosto de 2017 / Modificado: 2 de febrero de 2018 / Aceptado: 9 de febrero de 2018.

Para citar este artículo

Bonilla Ovallos, M. E. (2018). Propuesta de medición del desempeño policial en democracia. opera, 22, pp. 121-139. DOI: https://doi.org/10.18601/16578651.n22.07
} 


\section{INTRODUCCIÓN}

Desde sus orígenes la policía se ha constituido en una institución necesaria, relevante y funcional para la conservación del Estado y, por ende, su desempeño se ha vinculado estrechamente con el régimen político y con la forma en que se ha obtenido, ejercido y mantenido el poder a lo largo del tiempo. Sin embargo, pese a su papel protagónico es evidente el número limitado de estudios que existen acerca del tema en comparación con los que se han adelantado sobre otras instituciones políticas como los partidos, las asambleas legislativas, entre otras, lo cual hace pertinente y valioso enfocar esfuerzos en adelantar investigaciones que aborden el tema de policía y régimen político, sobre todo porque es evidente que en el escenario político actual la democracia convive con diversos grados de autoritarismo y abuso policial y, en términos generales, en muchos Estados es cuestionable el nivel de empoderamiento de los ideales democráticos en el desempeño de sus cuerpos de seguridad (Cruz, 2010).

De allí que el objetivo de este artículo se oriente a plantear un modelo de medición del desempeño policial en un régimen democrático -el Índice de Desempeño Policial Democrático (IDPD)-, que le permita a los países monitorear el reforzamiento, la afirmación y el robustecimiento de los principios elementales de la democracia dentro de sus cuerpos policiales y establecer su grado de coherencia y funcionalidad para la consolidación del régimen. Dicho propósito, si bien es pertinente en la medida en que abre caminos para la observación de una de las instituciones del Estado menos estudiada (Bayley, 1985; Tudela, 2011), no es para nada sencillo, puesto que pretende contribuir con elementos que de facto apuntan a un asunto de elevada complejidad, la construcción de fuerzas policiales democráticas, incorporando dimensiones y proponiendo indicadores de medición tanto de los resultados de la labor policial, como de las características propias que esta posee.

El artículo se encuentra estructurado en tres partes. En primer lugar, plantea reflexiones sobre las características que debe presentar el desempeño de un cuerpo policial coherente y funcional para la consolidación democrática. En segundo lugar, define las dimensiones y los indicadores que deben tenerse en cuenta para medir el desempeño de una institución policial que opere en escenarios democráticos, planteando criterios de clasificación para cada uno de ellos y proponiendo la construcción de un subíndice para cada dimensión. En tercer lugar, presenta el Índice de Desempeño Policial Democrático (IDPD) como un mecanismo de monitoreo de los niveles de desempeño policial en el marco de un régimen democrático.

\section{DESEMPEÑO POLICIAL Y CONSOLIDACIÓN DEMOCRÁTICA}

La consolidación democrática entendida como "el proceso de reforzamiento, afirmación, robustecimiento del sistema democrático" (Morlino, 2007, p. 13), requiere para su concreción que las estructuras, las normas, los ideales y los principios fundamentales el régimen, además de estar definidos constitucionalmente, se encuentren arraigados, materializados y sean el común denominador de las relaciones existentes entre las diversas instituciones 
del Estado y los miembros de la sociedad. En consecuencia, la consolidación democrática requiere instituciones eficientes en la reducción de los conflictos, que se encuentren limitadas, subordinadas y controladas por el poder civil, que operen en defensa del respeto de los principios y los valores democráticos, y que estén legitimadas ante la sociedad (Schedler, 1998; Dahl, 2002; Diamond, 1997).

Dentro del amplio conjunto de instituciones del Estado, la policía desempeña un papel fundamental en la medida en que sobre ella reside una parte importante del monopolio de la fuerza y del ejercicio de la coacción legítima, cuya instrumentalización persigue el objetivo de mantener en niveles aceptables el orden y la seguridad. De allí que valga la pena reflexionar en torno a ¿̇cuáles deben ser las características del desempeño de una institución policial coherente con los principios del sistema democrático y, por ende, favorable para su consolidación?

El establecimiento de un modelo de policía coherente con el sistema democrático plantea la necesidad de que el cuerpo policial se interrelacione con los diversos agentes del Estado, institucionales y civiles, dentro de esquemas de respeto a los derechos constitucionales y democráticos, de manera que sus procedimientos contribuyan a reafirmar y reforzar constantemente los principios intrínsecos del régimen, como la libertad, la igualdad y la justicia (Dammert, 2005).

En este sentido, la consolidación democrática está directamente relacionada con la forma en que el cuerpo de seguridad es capaz de interiorizar y afirmar los valores y las normas democráticas en la resolución de los distintos conflictos que puedan generarse en la sociedad, manteniendo ante todo su compromiso democrático y garantizando, con las características de su desempeño, un conjunto más amplio de derechos que favorezca su legitimidad y la del régimen.

Como bien se ha sostenido, "en un Estado democrático es necesaria la policía, una policía democrática" (Rico, 1983, p. 10), que progrese constantemente hacia su modernización, y que sea garante de los derechos ciudadanos "que forman parte integral del proceso democrático o son esenciales para él" (Dahl, 2002, p. 219). No obstante, en algunos casos es posible que la policía, a fin de maximizar el bienestar colectivo, transgreda ciertos intereses particulares debido a que generalmente "las decisiones vinculadas a los asuntos públicos son, en gran parte, decisiones sobre la distribución de los beneficios y los costos y las ganancias y los prejuicios" (p. 220).

$\mathrm{Al}$ ser "el uso de la fuerza física el rasgo más destacado de la actividad policial" (González, 2006, p. 17), la cual puede ser usada en nombre de la colectividad para prevenir y reprimir la violación de las reglas que rigen al grupo, es posible que sus efectivos enfrenten con bastante regularidad la dicotomía entre garantizar o vulnerar de manera transitoria los derechos reconocidos constitucionalmente, pues su contacto directo y constante con los ciudadanos, las víctimas, los victimarios y el crimen los hace propensos a incurrir en malas prácticas policiales durante el ejercicio de sus funciones.

De allí, que el uso de la fuerza policial sea "un problema preciso de gobernar" (Aimar et al., 2005, p. 16), dado que un desempeño policial caracterizado por el excesivo uso de la fuerza, el abuso de autoridad, la negligencia, 
la arbitrariedad y la corrupción terminaría por afectar la relación de cercanía y cooperación con la ciudadanía, el nivel de confianza en la institución y la percepción sobre su grado de eficiencia en el establecimiento de condiciones idóneas de seguridad, así como la vulneración de los principios fundamentales de la democracia (Ahmad, Hubickey y McNamara, 2011; Hales y Higgins, 2016).

Lo anterior implica un serio obstáculo para la consolidación del régimen político, pues en democracia existen unos "derechos o intereses considerados inviolables y por ende superiores al proceso democrático mismo" (Dahl, 2002, p. 220), que deben ser protegidos por las instituciones de seguridad y, en consecuencia, resulta inaceptable que el desempeño policial los transgreda. La consolidación democrática requiere la existencia de un cuerpo policial que, entre otras cosas, no utilice su principal potestad -el uso de la fuerza- de manera arbitraria, desigual, injusta y sesgada, ni se preste para la defensa de intereses políticos, ni sea instrumento para la violación de los derechos humanos y la dignidad humana.

En este sentido resulta inconsistente con la consolidación democrática que funcionarios policiales inflijan, instiguen o toleren actos de tortura, penas crueles, trato inhumano o degradante, invocando como justificación para la comisión de los mismos la orden de un superior o circunstancias especiales como estado de guerra, amenaza a la seguridad, inestabilidad política interna o cualquier otra emergencia pública. Por el contrario, "solamente deben utilizar las armas en las situaciones en que exista un riesgo racionalmente grave para su vida, su integridad física o la de terceras personas, o en aquellas circunstancias que puedan suponer un grave riesgo para la seguridad ciudadana” (Tudela, 2007, p. 17).

Sin embargo, no basta con garantizar que el desempeño policial sea respetuoso de los derechos humanos y del orden constitucional, y haga uso moderado y proporcional de la fuerza para que exista una policía favorable a la consolidación democrática. También es necesario que sea capaz de suplir las necesidades y demandas de los ciudadanos, pues el mantenimiento de la paz ciudadana tendrá repercusiones importantes sobre la forma en que estos resuelven sus diferencias y la credibilidad que tienen en la policía.

Si la policía, en un Estado democrático, se instrumentaliza en pos de reconocer, aumentar y garantizar los derechos y las obligaciones ciudadanas que se derivan del orden político, así como de proveer mayores niveles de seguridad, los diversos miembros de la sociedad reconocerán en el cuerpo de seguridad "the only game in town" (Linz y Stepan, 1996), es decir, la mejor opción para llevar a cabo sus acuerdos y resolver sus diferencias, considerando que la violación de las normas y las leyes vigentes desembocará en salidas costosas e ineficientes, ante lo cual, se sujetarán y habituarán a la resolución de los conflictos dentro de los límites que esta les impone.

En consecuencia, los problemas de seguridad ciudadana y convivencia que aquejan a la población introducen reflexiones pertinentes cuando comprendemos que las instituciones competentes deben proveer seguridad dentro del marco de los principios fundamentales del sistema democrático, entonces: ¿la policía debe proporcionar seguridad bajo qué 
circunstancias o condiciones?, ¿a qué costo?, ¿de qué forma?

Las exigencias actuales del Estado de derecho y de la consolidación democrática obligan a pensar en que el enfoque de la seguridad y las prácticas que se implementan para alcanzar los niveles requeridos por la sociedad están relacionados directamente con los conceptos de democracia y gobernabilidad, lo cual "configura un espacio sociopolítico determinante en las condiciones y la viabilidad de los esfuerzos" (Tudela, 2007, p. 3) que deben impulsar y ejecutar los gobiernos y la policía.

La democracia debe garantizar la seguridad, pero también la libertad, la igualdad y la justicia, y ninguno de estos valores fundamentales debe transarse por la consecución del otro. Por tanto, es vital que en el afán de proveer seguridad no se transgredan los derechos de las personas reconocidos constitucionalmente. Así pues, "la búsqueda de seguridad debe ser coherente y complementaria con los propósitos del Estado y de la democracia y este desafío demanda la adecuada interrelación de las políticas y la gestión de los gobiernos enmarcados e inspirados en una visión de seguridad en democracia" (López y Tudela, 2005, p. 22).

Actualmente, el objeto de la seguridad no es el Estado sino el ciudadano, a quien debe protegérsele y garantizársele sus derechos y sus libertades (Rico, 1983). De esta manera, la seguridad no es en sí misma un fin, por el contrario, es una garantía para el ejercicio de los derechos y el bien social, por lo cual es fundamental que la policía opere dentro del marco del Estado de derecho y evite la realización de prácticas que atenten contra su misión y la calidad de la democracia. Así, "la calidad de los servicios policiales y los modelos y estrategias empleados son clave, tanto desde una perspectiva sociopolítica (calidad de la democracia) como desde el punto de vista de las personas (inseguridad)" (Tudela, 2012, p. 381).

Resulta entonces fundamental para la consolidación democrática que las acciones de la institución policial se efectúen dentro de los parámetros del sistema democrático, lo cual "implica la supervisión del uso de los poderes policiales, no para que no se usen, sino para que se usen legítimamente" (López y Frühling, 2008, p. 7). La policía que opera en regímenes democráticos debería tener establecidos códigos de ética y de conducta para regular sus prácticas y enmarcarlas dentro del respeto de los derechos de los ciudadanos y el orden constitucional, con el objetivo de ser funcionales para el fortalecimiento del régimen $y$, en caso de que estos códigos sean vulnerados, los controles institucionales independientes y permanentes deben ser capaces de investigar y proponer la adopción de las medidas correctivas necesarias.

Para garantizar que el desempeño policial se adhiera a los principios democráticos es vital que la policía esté sometida a mecanismos de supervisión, internos y externos, que contribuyan a la consolidación de un desempeño policial más transparente, moderno y eficiente que "evite las extralimitaciones, los abusos y las arbitrariedades que no solamente impiden prevenir o conjurar aquellas conductas violatorias de derechos sino que, peor aún, se constituyen en una fuente de violaciones de derechos y por ende de cercenamientos y vulneraciones a la situación de seguridad pública" (Saín, 2003, pp. 2-3). 
El avance hacia una policía sujeta a las reglas de la ley y respetuosa de los derechos de las personas implica garantizar la responsabilidad de las conductas de la institución y de cada uno de sus representantes. Para ello, se requiere construir sistemas de responsabilización policial que impliquen la existencia de controles coherentes y complementarios y que requieren de la participación de distintas instituciones (López y Frühling, 2008, p. 8).

Sin embargo, es indispensable que aquellos quienes están a cargo de ejercer el control sobre el cuerpo policial también estén sometidos al proceso democrático.

En consecuencia, es posible afirmar que el desempeño de una institución policial coherente con los principios democráticos y favorable para la consolidación del régimen político debe estar caracterizado por tres aspectos clave: efectividad policial en la consecución de su misión institucional; legalidad policial en el ejercicio de sus funciones, y legitimidad policial, es decir, que cuente con la confianza, la credibilidad y el respaldo de la ciudadanía (Bonilla, 2015; 2016).

\section{MEDICIÓN DEL DESEMPEÑO POLICIAL EN DEMOCRACIA}

Teniendo en cuenta las demandas que el régimen democrático presenta a los cuerpos policiales, es posible diseñar un modelo de medición del desempeño policial en democracia soportado en las tres dimensiones mencionadas: efectividad, legalidad y legitimidad. A continuación se procederá al análisis de cada una de ellas proponiendo indicadores para su operacionalización y planteando criterios de clasificación para cada uno de ellos, de manera que pueda calcularse un subíndice para cada dimensión.

\section{Dimensión 1: efectividad policial}

La primera dimensión del desempeño policial democrático, efectividad policial, se centra en la calidad de la respuesta que el cuerpo policial ofrece a las demandas ciudadanas en torno a la seguridad y la convivencia, lo cual está estrechamente relacionado con su misión institucional, la cual radica en garantizar a la sociedad donde opera condiciones de paz ciudadana para prevenir, contener y combatir la criminalidad y la violencia en sus diversas manifestaciones.

Si bien, las variables que inciden en la seguridad y la convivencia son complejas y multicausales y, en consecuencia, demandan acciones de coordinación y responsabilidad interinstitucional, lo cierto es que ninguna otra institución del Estado tiene esta facultad tan claramente definida dentro de su misión, ni se establece como la cara visible de la seguridad pública ante la comunidad, como lo es la policía. En este sentido, pese a que los esquemas de seguridad pública de los países están integrados por diversas instituciones que prestan sus servicios en función de proveer más y mejor seguridad a la población, "la policía es uno de estos componentes y la calidad de su desempeño es muy relevante, pues constituye la cara visible e inmediata del Estado y de los esfuerzos gubernamentales en el marco de las políticas criminales" (Tudela, 2011, p. 5). 
Evaluar y medir esta dimensión resulta complejo por varias razones: 1) las problemáticas de inseguridad y violencia responden a variables de tipo multicausal en las que convergen dimensiones que se encuentran fuera de la competencia directa del cuerpo policial o que, en su defecto, demandan acciones de coordinación y responsabilidad interinstitucional; 2) los resultados positivos de la labor policial (outputs) son a menudo invisibles, en la medida en que "una buena policía es, en parte, una policía que logra que ciertos hechos no ocurran" (Cano, 2003, p. 3); 3) pese a tener clara la misión última de la institución en un contexto democrático, resulta inicuo diseñar un modelo que establezca las mismas exigencias en términos de eficiencia a los diversos cuerpos policiales que operan en contextos de seguridad y violencia disímiles y que cuentan con medios y capacidad institucional dispar, lo que puede justificar por sí mismo el establecimiento de metas distinta por atender e incluso incorporar concepciones de valor contrapuestas (Cano, 2003); 4) la calidad, confiabilidad y consistencia de los indicadores, además de verse afectados por el sesgo de estimación originado por la "cifra negra", pueden ser objeto de manipulación por parte de la propia institución, en la medida en que la policía es evaluada en función de lo que estos reflejan y cuanto más apreciable sea para ella la evaluación, mayor probabilidad de registrar datos poco fiables (Reiner, 1998); 5) las oscilaciones presentadas en los indicadores utilizados internacionalmente para medir la efectividad policial, como por ejemplo los homicidios, los robos y los hurtos, responden a un mayor número de condiciones causales ajenas a la institución, lo cual puede sesgar los resultados arrojados por el modelo de medición.

No obstante, pese a las dificultades mencionadas, los indicadores utilizados para medir la dimensión de efectividad policial están determinados por la evolución de los delitos y las contravenciones, los cuales pueden monitorearse mediante los registros estadísticos generados por las entidades competentes como la policía, medicina legal y los observatorios de violencia. Asimismo, las encuestas de percepción ciudadana se establecen como una fuente importante de información para tal fin, específicamente las preguntas que indagan sobre el grado de victimización de la población. Sin embargo, dada la distribución georreferencial asimétrica del delito y las contravenciones presentes entre los países y al interior de estos, es necesario seleccionar una canasta de indicadores por utilizar que refleje la realidad de la criminalidad de los países objeto de estudio.

A continuación se presenta una lista de indicadores que pueden ser utilizados para medir la eficiencia del desempeño policial en el control de la criminalidad y la violencia en contextos democráticos. De igual forma, se propone un criterio de clasificación para cada uno de ellos, que oscila entre 0 y 1 , donde 0 corresponde a una situación ideal para el delito y las contravenciones, y 1 representa el "peor escenario posible" (tabla 1). A fin de definir los criterios de clasificación propuestos para cada indicador se acudió a la clasificación realizada por la Organización Mundial de la Salud (oms) en el caso de los homicidios y, para el resto de indicadores seleccionados para medir 
esta dimensión, se revisaron las tasas más altas de delitos registrados nivel mundial en países que definen su régimen como una democracia, para establecer cuatro rangos de efectividad.

Para la medición de la efectividad policial $(E P)$ se propone agregar, mediante el método del promedio simple, cada uno de los valores obtenidos por los indicadores de acuerdo al criterio de clasificación propuesto, de manera que se obtenga un valor agregado que oscile entre 0 y 1 , el cual puede clasificarse en las siguientes cuatro categorías: 1) efectividad policial ideal, cuando la EP $=0$, lo que implica que la paz ciudadana no se ve perturbada por ninguna acción delictiva; 2) efectividad policial alta, cuando $0>\mathrm{EP} \leq 0,33$, lo que representa una tasa aceptable de delitos y contravenciones por cada 100 mil habitantes; 3) efectividad policial media, cuando $0,33>\mathrm{EP} \leq 0,66$, lo cual corresponde a una tasa de delitos y contraven-

TABLA 1. INDICADORES DE EFECTIVIDAD POLICIAL

\begin{tabular}{|c|c|c|}
\hline Indicadores & Fuentes & Criterio de clasificación y valor del indicador \\
\hline $\begin{array}{l}\text { Tasa anual de homicidios por cada } \\
100 \text { mil habitantes }\end{array}$ & $\begin{array}{l}\text { Institución policial } \\
\text { Medicina legal } \\
\text { Observatorios del delito }\end{array}$ & $\begin{array}{l}=0 \text { Efectividad policial ideal }=0 \\
\geq 1 \leq 10 \text { Efectividad policial alta }=0,33 \\
>10 \leq 20 \text { Efectividad policial media }=0,66 \\
>20 \text { Efectividad policial baja }=1\end{array}$ \\
\hline $\begin{array}{l}\text { Tasa anual de hurtos y robos por } \\
\text { cada } 100 \text { mil habitantes }\end{array}$ & $\begin{array}{l}\text { Institución policial } \\
\text { Medicina legal } \\
\text { Observatorios del delito }\end{array}$ & $\begin{array}{l}=0 \text { Efectividad policial ideal }=0 \\
\geq 1 \leq 88 \text { Efectividad policial alta }=0,33 \\
>88 \leq 214 \text { Efectividad policial media }=0,66 \\
>214 \text { Efectividad policial baja }=1\end{array}$ \\
\hline $\begin{array}{l}\text { Tasa anual de lesiones personales } \\
\text { por cada } 100 \text { mil habitantes }\end{array}$ & $\begin{array}{l}\text { Institución policial } \\
\text { Medicina legal } \\
\text { Observatorios del delito }\end{array}$ & $\begin{array}{l}=0 \text { Efectividad policial ideal }=0 \\
\geq 1 \leq 88 \text { Efectividad policial alta }=0,33 \\
>88 \leq 214 \text { Efectividad policial media }=0,66 \\
>214 \text { Efectividad policial baja }=1\end{array}$ \\
\hline $\begin{array}{l}\text { Tasa anual de extorsiones por } \\
\text { cada } 100 \text { mil habitantes }\end{array}$ & $\begin{array}{l}\text { Institución policial } \\
\text { Medicina legal } \\
\text { Observatorios del delito }\end{array}$ & $\begin{array}{l}=0 \text { Efectividad policial ideal }=0 \\
\geq 1 \leq 44 \text { Efectividad policial alta }=0,33 \\
>44 \leq 107 \text { Efectividad policial media }=0,66 \\
>107 \text { Efectividad policial baja }=1\end{array}$ \\
\hline $\begin{array}{l}\text { Tasa anual de secuestros por cada } \\
100 \text { mil habitantes }\end{array}$ & $\begin{array}{l}\text { Institución policial } \\
\text { Medicina legal } \\
\text { Observatorios del delito }\end{array}$ & $\begin{array}{l}=0 \text { Efectividad policial ideal }=0 \\
\geq 0,001 \leq 0,20 \text { Efectividad policial alta }=0,33 \\
>0,20 \leq 0,50 \text { Efectividad policial media }=0,66 \\
>0,50 \text { Efectividad policial baja }=1\end{array}$ \\
\hline $\begin{array}{l}\text { Porcentaje de población que ha } \\
\text { sido víctima de un delito }\end{array}$ & Encuestas de victimización & $\begin{array}{l}=0 \% \text { Efectividad policial ideal }=0 \\
\geq 1 \% \leq 10 \% \text { Efectividad policial alta }=0,33 \\
>10 \% \leq 35 \% \text { Efectividad policial media }=0,66 \\
>35 \% \text { Efectividad policial baja }=1\end{array}$ \\
\hline $\begin{array}{l}\text { Porcentaje de personas encon- } \\
\text { tradas frente a total de personas } \\
\text { desaparecidas }\end{array}$ & $\begin{array}{l}\text { Institución policial } \\
\text { Medicina legal } \\
\text { Observatorios del delito }\end{array}$ & $\begin{array}{l}=100 \% \text { Efectividad policial ideal }=0 \\
\geq 70 \% \leq 99 \% \text { Efectividad policial alta }=0,33 \\
>30 \%<70 \% \text { Efectividad policial media }=0,66 \\
<30 \% \text { Efectividad policial baja }=1\end{array}$ \\
\hline
\end{tabular}

Fuente: elaboración propia 
ciones que no puede considerarse aceptable en democracia pero tampoco muy mala; 4) efectividad policial baja, cuando $0,66>\mathrm{EP} \leq 1$, lo que indica condiciones de seguridad extremadamente deterioradas, que afectan de manera significativa y alarmante el desarrollo de la vida en sociedad y el ejercicio pleno de los derechos ciudadanos.

\section{Dimensión 2: legalidad policial}

La segunda dimensión del desempeño policial democrático, legalidad policial, evalúa la forma en que las prácticas policiales se ajustan o no a los lineamientos de deontología ${ }^{1}$ policial, $y$ si las acciones de los miembros de la institución se desarrollan en función del respeto o la transgresión de los códigos de conducta y disciplina internos y externos, el cumplimento de la constitución y la ley, el respeto por la dignidad de las personas y la protección de los derechos humanos. Los indicadores utilizados para medir esta dimensión están relacionados con los procesos de corrupción y vinculación de policías con el delito y las contravenciones, así como las faltas disciplinarias simples o agravadas en las que estos puedan incurrir.

Como hemos indicado, una policía democrática debe proveer mayores niveles de seguridad y convivencia ciudadana dentro del marco de los principios fundamentales del sistema político (Saín, 2003; Tudela, 2007;
Bayley, 1985). Lo anterior "implica que debe garantizar la seguridad pero también la libertad, la igualdad y la justicia y ninguno de estos valores deben transarse por la consecución del otro. Por tanto, es vital que en el afán de proveer seguridad los efectivos policiales no transgredan los derechos de las personas reconocidos constitucionalmente" (Bonilla, 2015, p. 109).

En la democracia es necesario fortalecer la visión del tipo de seguridad que la policía debe proveer, puesto que su consecución "debe ser coherente y complementaria con los propósitos del Estado y del sistema político y este desafío demanda la adecuada interrelación de las políticas y la gestión de los gobiernos enmarcados e inspirados en una visión de seguridad en democracia" (López y Tudela, 2007, p. 22). Es decir, el desempeño policial no debe evaluarse "solo desde la perspectiva del resultado final, sino de la forma cómo actúa y cumple su misión" (Tudela, 2011, p. 33). De esta manera, la misión policial debe garantizarse soportando sus prácticas y estrategias ante todo en el respeto y la reivindicación de los lineamientos constitucionales y democráticos.

De allí que prácticas como la corrupción, el uso excesivo e injustificado de la fuerza, la arbitrariedad, el abuso de autoridad o la violación de los derechos de los ciudadanos, entre otras, intenten ser reguladas, prohibidas, controladas y condenadas desde el establecimiento

\footnotetext{
1 “El término 'deontología' proviene de los vocablos griegos deon, que significa deber, y logos, razonamiento o ciencia. Es una palabra que por primera vez utilizó el economista, jurista, literario y filósofo inglés Jeremías Bentham (1748-1832), en una corriente doctrinaria llamada utilitarismo, que versa sobre los deberes del ser humano, determinados de alguna manera por la ética y la moral, aplicadas a diversas situaciones sociales, es decir, el bien y el mal que cada persona tiene consigo mismo y con los que le rodean en la profesión que desempeña” (Herrera, 2006, p. 1).
} 
de códigos de ética y de conducta fundados en el respeto de los derechos de los ciudadanos y el orden constitucional, que pretenden, en últimas, desligar las malas prácticas del trabajo policial (Garriga, 2016).

Evaluar y medir la segunda dimensión del desempeño policial, la legalidad policial, impone retos relacionados principalmente con la obtención de información completa y fiable. En escenarios de corrupción, y cuando los mecanismos de control especializados están al interior de la propia policía, la situación es aún más compleja, pues al igual que en el caso de la efectividad policial, los indicadores pueden ser manipulados y alterados por los intereses propios de la institución, así como por la cifra negra, es decir, por el porcentaje de malas prácticas que no se denuncian, bien sea por desconfianza y temor de la ciudadanía frente a los nexos de los efectivos con el delito, o por el temor a las represalias de las que puedan ser objeto algunos efectivos, cuando su testimonio relaciona a un mando superior con transgresiones graves al código de conducta. Es allí donde "las encuestas de percepción ciudadana son nuevamente un buen instrumento, así como los datos y registros que pueden ser aportados por organismos de derechos humanos que realizan supervisión sobre las policías" (Mohor, 2007, p. 6).

Sin embargo, a pesar a las deficiencias señaladas anteriormente, los indicadores utilizados para medir la dimensión de legalidad policial están determinados por la cuantía en que los efectivos de la institución incurren en transgresiones a los códigos de conducta $y$, en general, permean los derechos ciudadanos reconocidos constitucionalmente. Dichas prácticas pueden monitorearse mediante los registros estadísticos generados por las entidades competentes como las oficinas de control interno y externo de la Policía, inspecciones, procuradurías, fiscalías, personerías y organizaciones no gubernamentales (ONG). Asimismo, las encuestas de percepción ciudadana se pueden establecer como fuente útil, específicamente cuando indagan sobre actos de corrupción propiciados por el cuerpo de seguridad.

A continuación se presenta una lista de indicadores que pueden ser utilizados para medir la legalidad policial en el ejercicio de sus funciones. Asimismo, se propone un criterio de clasificación para cada uno de ellos, que oscila entre 0 y 1 , donde 0 corresponde a una situación ideal de apego a la ley en las prácticas policiales y 1 representa el "peor escenario posible" de ilegalidad policial (tabla 2). Para definir los criterios de clasificación propuestos para cada indicador se acudió a la socialización y el debate sobre estos con funcionarios de la institución policial de tres países latinoamericanos: El Salvador, Colombia y Venezuela. Es importante señalar que ante el sesgo que puede generar la utilización exclusiva de las fuentes oficiales y la necesidad de contrastar la información con otras organizaciones, se recomienda utilizar todos los datos disponibles que al respecto se hayan generado en cada país, como los que se derivan de las encuestas de percepción, las ONG defensoras de derechos humanos y las denuncias periodísticas sobre abuso policial.

Para la medición de la legalidad policial (LP) se propone agregar, mediante el método del promedio simple, cada uno de los valores obtenidos por los indicadores de acuerdo con el 
TABLA 2. INDICADORES DE LEGITIMIDAD POLICIAL

\begin{tabular}{|c|c|c|}
\hline Indicadores & Fuentes & Criterio de clasificación y valor del indicador \\
\hline $\begin{array}{l}\text { Tasa anual de denuncias de abuso de autori- } \\
\text { dad policial por cada } 10 \text { mil policías }\end{array}$ & $\begin{array}{l}\text { Institución policial } \\
\text { Inspecciones } \\
\text { Procuradurías } \\
\text { ONG }\end{array}$ & $\begin{array}{l}\geq 0 \leq 0,99 \text { prácticas policiales ideales }=0 \\
>1 \leq 10 \text { buenas prácticas policiales }=0,33 \\
>10 \leq 20 \text { regulares prácticas policiales }=0,66 \\
>20 \text { malas prácticas policiales }=1\end{array}$ \\
\hline $\begin{array}{l}\text { Porcentaje de manifestantes heridos por } \\
\text { policías respecto del total de manifestantes } \\
\text { detenidos }\end{array}$ & $\begin{array}{l}\text { Institución policial } \\
\text { Medicina legal } \\
\text { Inspecciones } \\
\text { Procuradurías } \\
\text { ONG }\end{array}$ & $\begin{array}{l}=0 \% \text { prácticas policiales ideales }=0 \\
>1 \% \leq 2 \% \text { buenas prácticas policiales }=0,33 \\
>2 \leq 5 \text { regulares prácticas policiales }=0,66 \\
>5 \text { malas prácticas policiales }=1\end{array}$ \\
\hline $\begin{array}{l}\text { Porcentaje de población a la que se le ha } \\
\text { solicitado un soborno }\end{array}$ & $\begin{array}{l}\text { Encuestas de percepción } \\
\text { ciudadana }\end{array}$ & $\begin{array}{l}=0 \% \text { prácticas policiales ideales }=0 \\
>1 \% \leq 2 \% \text { buenas prácticas policiales }=0,33 \\
>2 \leq 5 \text { regulares prácticas policiales }=0,66 \\
>5 \text { malas prácticas policiales }=1\end{array}$ \\
\hline $\begin{array}{l}\text { Tasa anual de efectivos involucrados en si- } \\
\text { tuaciones en las que se generó muerte por } \\
\text { cada } 10 \text { mil policías }\end{array}$ & $\begin{array}{l}\text { Institución policial } \\
\text { Medicina legal } \\
\text { Inspecciones } \\
\text { Procuradurías } \\
\text { ONG }\end{array}$ & $\begin{array}{l}\geq 0 \leq 0,99 \text { prácticas policiales ideales }=0 \\
>1 \leq 10 \text { buenas prácticas policiales }=0,33 \\
>10 \leq 20 \text { regulares prácticas policiales }=0,66 \\
>20 \text { malas prácticas policiales }=1\end{array}$ \\
\hline $\begin{array}{l}\text { Tasa anual de efectivos sancionados, des- } \\
\text { tituidos, suspendidos o judicializados por } \\
\text { faltas al servicio, negligencia y por pertene- } \\
\text { cer a organizaciones al margen de la ley por } \\
\text { cada } 10 \text { mil policías (discriminado por rango } \\
\text { jerárquico, tipo de falta y género) }\end{array}$ & $\begin{array}{l}\text { Institución policial } \\
\text { Medicina legal } \\
\text { Inspecciones } \\
\text { Procuradurías } \\
\text { ONG }\end{array}$ & $\begin{array}{l}\geq 0 \leq 0,99 \text { prácticas policiales ideales }=0 \\
>1 \leq 10 \text { buenas prácticas policiales }=0,33 \\
>10 \leq 20 \text { regulares prácticas policiales }=0,66 \\
>20 \text { malas prácticas policiales }=1\end{array}$ \\
\hline $\begin{array}{l}\text { Porcentaje de presos que fueron miembros } \\
\text { de la policía e incurrieron en la transgresión } \\
\text { siendo miembros activos de la institución }\end{array}$ & Sistemas penitenciarios & $\begin{array}{l}=0 \% \text { prácticas policiales ideales }=0 \\
>1 \% \leq 10 \% \text { buenas prácticas policia- } \\
\text { les }=0,33 \\
>10 \% \leq 40 \% \text { regulares prácticas policia- } \\
\text { les }=0,66 \\
>40 \% \text { malas prácticas policiales }=1\end{array}$ \\
\hline
\end{tabular}

fuente: elaboración propia

criterio de clasificación propuesto, de manera que se obtenga un valor agregado que oscile entre 0 y 1 , el cual puede clasificarse en las siguientes cuatro categorías: 1) legalidad policial ideal, cuando la $\mathrm{LP}=0$, lo que corresponde a la ausencia total de malas prácticas policiales, es decir, a una institución completamente transparente, integral y respetuosa del marco normativo en el que opera; 2 ) legalidad policial alta, cuando $0>\mathrm{LP} \leq 0,33$, lo que representa una tasa moderada de corrupción, vinculación con el delito y faltas disciplinarias al interior de la policía; 3) legalidad policial media, cuando $0,33>\mathrm{LP} \leq 0,66$, lo que implica una tasa de transgresión de códigos de conducta y disciplinarios que no puede considerarse aceptable en una democracia, pero tampoco muy es mala; 4) legalidad policial baja, cuando $0,66>$ LP $\leq 1$, 
lo que indica una tasa de malas prácticas policiales extremadamente elevada, que hace del cuerpo de seguridad un nicho de corrupción y fuente de violencia y vulneración de derechos, que en lugar de proteger a los ciudadanos garantizándoles mayores y mejores niveles de bienestar, se instituye como un victimario más.

\section{Dimensión 3: legitimidad policial}

La tercera dimensión del desempeño policial democrático es la legitimidad policial, la cual evalúa los niveles de confianza, credibilidad y respeto que los ciudadanos declaran tener en la policía. Los indicadores planteados para medir esta dimensión están relacionados con la percepción de los miembros de la sociedad en la que el cuerpo de seguridad opera, de allí que las encuestas de percepción ciudadana sean la fuente principal de información para conocer tal fin, en especial aquellas preguntas que indagan sobre la calidad del servicio que el cuerpo de seguridad presta, la confianza que los ciudadanos depositan en la policía, el trato que reciben de su parte y la sensación de seguridad (o inseguridad) que les genera la institución.

En democracia, al ser el ciudadano el cliente de la gestión policial la percepción que este tenga de ella es determinante a la hora de evaluar su función, puesto que es indispensable que la institución de seguridad cuente con el respeto y el reconocimiento de la ciudadanía, sea acreedora de su confianza y se encuentre exaltada por su trato respetuoso, atento y diligente Lo anterior es fundamental para que "las organizaciones policiales puedan llevar a cabo su actividad cotidiana y perdurar en el tiempo" (Requena, 2016, p. 3). Asimismo, es la comunidad la que puede dar fe del grado de acercamiento que esta institución ha establecido con la sociedad y, a manera de percepción, evaluar los modelos de prestación del servicio policial fundados en la vocación de trabajo comunitario y de proximidad.

De igual forma, la legitimidad social de la acción policial es clave porque ella se asocia a la gobernabilidad, es decir, al derecho a gobernar y al reconocimiento de ese derecho por parte de los gobernados (Beetham, 1991). En este sentido, haber sido víctima del crimen erosiona el compromiso de los ciudadanos con el respeto de la ley por parte de la policía. Así, en la medida en que las personas se sienten más inseguras, están más dispuestas a aprobar que la policía actúe al margen de la ley con tal de atrapar delincuentes, y con ello se erosiona el Estado de derecho y se elevan las demandas ciudadanas en torno a un desempeño policial más efectivo en la lucha contra el delito y las contravenciones (Seligson y Smith, 2010).

En consecuencia,

...a mayor legitimidad de la policía, mayor disposición por parte de la ciudadanía a aceptar y a cumplir sus órdenes, a colaborar activamente con ella y a cumplir con las leyes en general. Por el contrario, a menor legitimidad policial, genera menor deferencia hacia la policía y sus agentes, menor colaboración y predisposición al cumplimiento voluntario de las normas, lo que conduce a escenarios de gobernabilidad en los que predominan las intervenciones policiales coactivas (Requena, 2016, p. 5).

De allí que la obtención del objetivo de promover la legitimidad del cuerpo de seguridad resulte clave a la hora de evaluar el desem- 
peño policial dentro del marco democrático, no solo porque las reformas policiales puestas en marcha a finales del siglo Xx en países gobernados por este régimen así lo declararán, sino porque esta resulta clave para estrechar la conexión entre los individuos y las estructuras sociales y políticas, favoreciendo la consolidación del sistema político.

De esta manera, la legitimidad policial podría catalogarse como el producto de un proceso

...sometido a la experiencia y a las valoraciones que las personas hacen del funcionamiento cotidiano de la policía [...], lo que supone afirmar que la ciudadanía se siente obligada a obedecerla, a seguir las indicaciones de sus agentes, de quienes piensan que actúan de acuerdo con lo que establecen las leyes y las normas que regulan la función policial, y con quienes comparten buena parte de los valores morales dominantes en la sociedad de referencia (Requena, 2016, p. 11).

De allí que esta dimensión haga referencia a la capacidad de la policía para obtener obediencia, respeto y reconocimiento por parte de la población, sin necesidad de recurrir al uso extremo y arbitrario de la fuerza, de tal forma que los ciudadanos sean capaces de aceptar y reconocer su autoridad porque la ética del ejercicio policial y la ejemplaridad de su accionar legitiman políticamente su poder (Jackson, Brandford y Stanko, 2012; Hough, Jackson y Brandford, 2010).

$\mathrm{Al}$ igual que en el caso de la efectividad policial y las prácticas policiales, los indicadores propuestos para la medición de esta dimensión presentan limitaciones debido a que las fuentes principales son las encuestas de percepción, las cuales están afectadas por sesgos de orden social, cultural, psicológico e histórico que influyen en la percepción de los ciudadanos, así como por los sesgos propios del diseño metodológico como son las características del muestreo, el orden de las preguntas, las particularidades del trabajo de campo, los criterios de codificación y los margenes de error (PNUD, 2013).

No obstante las propias limitaciones de los indicadores señaladas, se presenta una lista de indicadores que pueden ser utilizados para medir la legitimidad policial en democracia. Asimismo, se propone un criterio de clasificación para cada uno de ellos que oscila entre 0 y 1 , donde 0 corresponde a los mejores niveles de legitimidad de los que pueda gozar el cuerpo de seguridad, es decir, que todos los ciudadanos de la circunscripción específica donde opera declaran confiar, creer y respetar a la policía, y 1 representa el peor escenario posible de ausencia de legalidad policial (tabla 3). Para definir los criterios de clasificación propuestos para cada indicador de esta dimensión del desempeño policial democrático se acudió a la socialización y el debate sobre estos con funcionarios de la institución policial de tres países latinoamericanos: El Salvador, Colombia y Venezuela.

Para la medición de la legitimidad policial (LEP) se propone agregar, mediante el método del promedio simple, cada uno de los valores obtenidos por los indicadores de acuerdo al criterio de clasificación propuesto anteriormente, de manera que se obtenga un valor agregado que oscile entre 0 y 1 , el cual puede clasificarse en las siguientes cuatro categorías: 1) legitimidad policial ideal, cuando la LEP $=0$, lo que corresponde a una institución que 
TABLA 3. INDICADORES DE LEGITIMIDAD POLICIAL

\begin{tabular}{|c|c|c|}
\hline Indicador & Fuentes & Criterio de clasificación y valor del indicador \\
\hline $\begin{array}{l}\text { Porcentaje de ciudadanos que confía en } \\
\text { la policía respecto a la población total }\end{array}$ & $\begin{array}{l}\text { Encuestas generales de } \\
\text { percepción ciudadana }\end{array}$ & $\begin{array}{l}=100 \% \text { legitimidad policial ideal }=0 \\
>80 \% \leq 99 \% \text { legitimidad policial alta }=0,33 \\
>50 \% \leq 80 \% \text { legitimidad policial media }=0,66 \\
\leq 50 \% \text { legitimidad policial ideal }=1\end{array}$ \\
\hline $\begin{array}{l}\text { Porcentaje de ciudadanos que evalúa } \\
\text { bien o muy bien la actuación policial } \\
\text { en prevención de delitos respecto a la } \\
\text { población total }\end{array}$ & $\begin{array}{l}\text { Encuestas generales de } \\
\text { percepción ciudadana }\end{array}$ & $\begin{array}{l}=100 \% \text { legitimidad policial ideal }=0 \\
>80 \% \leq 99 \% \text { legitimidad policial alta }=0,33 \\
>50 \% \leq 80 \% \text { legitimidad policial media }=0,66 \\
\leq 50 \% \text { legitimidad policial baja }=1\end{array}$ \\
\hline $\begin{array}{l}\text { Porcentaje de ciudadanos que consi- } \\
\text { dera que la policía es nada o muy poco } \\
\text { corrupta respecto a la población total }\end{array}$ & $\begin{array}{l}\text { Encuestas generales de } \\
\text { percepción ciudadana }\end{array}$ & $\begin{array}{l}=100 \% \text { legitimidad policial ideal }=0 \\
>80 \% \leq 99 \% \text { legitimidad policial alta }=0,33 \\
>50 \% \leq 80 \% \text { legitimidad policial media }=0,66 \\
\leq 50 \% \text { legitimidad policial baja }=1\end{array}$ \\
\hline $\begin{array}{l}\text { Porcentaje de ciudadanos que consi- } \\
\text { dera que la policía no está involucrada } \\
\text { en actividades delictivas respecto a la } \\
\text { población total }\end{array}$ & $\begin{array}{l}\text { Encuestas generales de } \\
\text { percepción ciudadana }\end{array}$ & $\begin{array}{l}=100 \% \text { legitimidad policial ideal }=0 \\
>80 \% \leq 99 \% \text { legitimidad policial alta }=0,33 \\
>50 \% \leq 80 \% \text { legitimidad policial media }=0,66 \\
\leq 50 \% \text { legitimidad policial baja }=1\end{array}$ \\
\hline $\begin{array}{l}\text { Porcentaje de ciudadanos que considera } \\
\text { que la problemática de inseguridad ha } \\
\text { mejorado con respecto al año anterior }\end{array}$ & $\begin{array}{l}\text { Encuestas generales de } \\
\text { percepción ciudadana }\end{array}$ & $\begin{array}{l}=100 \% \text { legitimidad policial ideal }=0 \\
>80 \% \leq 99 \% \text { legitimidad policial alta }=0,33 \\
>50 \% \leq 80 \% \text { legitimidad policial media }=0,66 \\
\leq 50 \% \text { legitimidad policial baja }=1\end{array}$ \\
\hline $\begin{array}{l}\text { Porcentaje de ciudadanos que consi- } \\
\text { dera que, comparado con el año ante- } \\
\text { rior, la policía ha sido más efectiva }\end{array}$ & $\begin{array}{l}\text { Encuestas generales de } \\
\text { percepción ciudadana }\end{array}$ & $\begin{array}{l}=100 \% \text { legitimidad policial ideal }=0 \\
>80 \% \leq 99 \% \text { legitimidad policial alta }=0,33 \\
>50 \% \leq 80 \% \text { legitimidad policial media }=0,66 \\
\leq 50 \% \text { legitimidad policial baja }=1\end{array}$ \\
\hline
\end{tabular}

Fuente: elaboración propia

cuenta con la confianza, el respeto y la credibilidad de todos los usuarios de su servicio: los ciudadanos; 2) legitimidad policial alta, cuando $0>\operatorname{LEP} \leq 0,33$, lo que hace referencia a un porcentaje elevado de ciudadanos que confía en la policía y tiene una percepción positiva sobre su desempeño; 3) legitimidad policial media, cuando $0,33>\operatorname{LEP} \leq 0,66$, lo que corresponde un porcentaje de ciudadanos que desconfía, no cree, ni respeta a la policía, que no puede considerarse aceptable en democracia pero tampoco es muy malo; 4) legi- timidad policial baja, cuando $0,66>\operatorname{LEP} \leq 1$, lo que indica un porcentaje muy elevado de ciudadanos frente a los cuales la institución policial se encuentra deslegitimada.

\section{ÍNDICE DE DESEMPEÑO POLICIAL DEMOCRÁTICO (IDPD)}

Después de establecer lo que se entiende por desempeño policial democrático y proponer las dimensiones y los indicadores a través 
de los cuales se puede medir, este apartado presenta un modelo para su medición, el Índice de Desempeño Policial Democrático (IDPD), que permite a los países que operan dentro de esquemas democráticos monitorear el reforzamiento, la afirmación y el robustecimiento de los principios elementales de la democracia dentro de sus cuerpos policiales, de manera que se instituye como un instrumento para monitorear los avances o retrocesos en el grado de democratización que la institución presenta a lo largo del tiempo.

El IDPD se diseña como una propuesta de medición que agrega las diversas dimensiones del desempeño policial y la multiplicidad de indicadores que pueden ser útiles para su operacionalización. En este sentido, la amplia información que implica analizar el desempeño policial hace necesario buscar una metodología integradora que pueda ser utilizada por los entes de control para realizar un adecuado seguimiento y evaluación del desempeño de su cuerpo de seguridad y determinar su grado de funcionalidad para la consolidación del régimen, al establecerse como un indicador compuesto que recoge información de tres dimensiones concretas: efectividad, legalidad y legitimidad policiales.

Este índice se calcula con base en el promedio ponderado obtenido por cada una de las tres dimensiones que se van a evaluar, el cual podrá oscilar entre una escala de 0 a 1 . Los valores obtenidos permitirán clasificar el desempeño policial democrático en cuatro categorías: 1) ideal $(\operatorname{IDPD}=0)$; 2) alto $(0>\operatorname{IDPD} \leq 0,33)$; 3) medio $(0,33>$ IDPD $\leq 0,66)$; 4) bajo $(0,66>$ IDPD $\leq 1)$. Donde el IDPD ideal representa un desempeño absolutamente demo- crático, el alto representan un desempeño policial más coherente con los principios y fundamentos democráticos $\mathrm{y}$, por ende, favorable para la consolidación del régimen y, por el contrario, el IDPD bajo indica un desempeño policial menos funcional para la consolidación democrática.

Para el cálculo de IDPD se utiliza la siguiente ecuación que agrega los subíndices construidos para cada dimensión, teniendo en cuenta el siguiente peso porcentual: $35 \%$ efectividad policial, $35 \%$ legalidad policial y $30 \%$ legitimidad policial:

$$
I D P D=\sum_{i=1}^{4} \beta_{i}\left(D_{i}\right)
$$

Donde:

$\beta$ es la importancia relativa de cada subíndice $(D)$ con respecto a las tres dimensiones.

Por su parte, para el cálculo de los subíndices de cada una de las tres dimensiones se utiliza la siguiente ecuación:

$$
D=\sum_{i=1}^{N} \beta_{i}\left(I_{i}\right)
$$

Donde:

- I es el indicador.

- $N$ es el número de indicadores por usar para la estimación.

- $\beta$ es la importancia relativa de cada indicador con respecto al total de indicadores.

Es importante aclarar que para ambos ejercicios estimativos: cálculo del IDPD y de los subíndices de cada dimensión, debe tenerse en cuenta que la suma de los $\beta_{i}$ sea igual a la uni$\mathrm{dad}$, es decir, debe ponderarse la importancia 
de cada dimensión $(D)$ o de cada indicador $(I)$, respecto del conjunto que los engloba, esto en función del contexto particular en el que opera cada cuerpo policial y las metas institucionales enmarcadas en la formulación de políticas públicas.

\section{REFLEXIONES FINALES}

Medir y evaluar el desempeño de la policía implica enfrentar una multiplicidad de obstáculos dentro de los que se destacan: 1) la ausencia de enfoques comunes en torno a lo que se entiende por buen desempeño policial, lo cual ha imposibilitado el desarrollo de herramientas de monitoreo y medición, puesto que existen "diversas miradas, aproximaciones, expectativas, niveles de análisis, modelos de policía y escasos datos" (Tudela, 2011, p. 5); 2) la "resistencia al escrutinio de cualquier mirada externa que es un rasgo común en policías de diferentes tradiciones y contextos culturales, que subsiste" (p. 8), debido a la débil tradición de análisis respecto al rol social de la policía, a sus elevados niveles de secretismo y al sesgo ideológico que usualmente caracteriza a los estudios sobre esta institución (Jar, 1999; Bayley, 1985);3) las limitaciones propias que enfrenta cualquier indicador cuantitativo en función de la metodología usada para la recolección y la sistematización de los datos, que en el caso de los indicadores de desempeño policial pueden resultar aún más imprecisos, debido al sesgo de estimación originado por la "cifra negra" o el subregistro ${ }^{2}$ que se deriva, entre otras cosas, de la relación costo-beneficio de interponer denuncias, de los acuerdos y procesos informales que se dan entre las partes para reivindicar, reparar y resolver conflictos, y por el hecho de que algunos delitos carecen de víctimas directas, como por el ejemplo el tráfico de drogas, de armas y el contrabando (Cano, 2003); 4) la limitada posibilidad de contraste de fuentes de información, como consecuencia de que la generación de indicadores de desempeño policial usualmente está bajo la responsabilidad del mismo cuerpo de seguridad y responde a las pretensiones de recolección, sistematización y divulgación de datos que esta se trace.

No obstante, y pese a que en las democracias modernas la policía es "un objeto opaco cuyos esfuerzos por estudiarla y comprenderla solo pueden ser calificados como parcialmente satisfactorios" (Gónzalez, Monteri y Sozzo, 2005, p. 7), vale la pena adelantar esfuerzos al respecto, dada su funcionalidad para la institución, los organismos de gobierno, la sociedad y el mismo régimen, pues, como lo sostienen diversos autores, aquellos cuerpos policiales que se han sometido a procesos serios, rigurosos y externos de evaluación, han presentado mayores avances en términos de profesionalización y democratización (Frühling, 2001).

Asimismo, el esfuerzo por proponer esquemas de medición de esta índole resulta novedoso puesto que son pocos los investigadores y las instituciones que en el mundo, y particularmente en América Latina, se han encargado de trabajar el tema, pese a que la reforma policial fue una de las tantas transformaciones institucionales llevadas a cabo en

2 Número de denuncias que no se realizan sobre el total de crímenes o abusos reales. 
diversos países a finales del siglo pasado. Si bien el estudio sobre la implementación y los efectos de las reformas se ha abordado, existe un claro déficit investigativo sobre la relación de estas con los niveles de calidad y consolidación de la democracia.

\section{REFERENCIAS}

Ahmad, N., Hubickey, V. y McNamar, A. (2011). La confianza en la policía nacional. Perspectivas desde el barómetro de las Américas, (59). Recuperado de https://www.vanderbilt.edu/lapop/insights/ I0859es.pdf

Aimar, V., González, G., Montero, A. y Sozzo, M. (2005). Política, policía y violencia en la provincia de Santa Fe". En M. Sozzo (dir.), Policía, violencia, democracia. Ensayos sociológicos (pp. 15-63). Santa Fe: Universidad Nacional del Litoral, Facultad de Ciencias Jurídicas y Sociales.

Alcántara, M. (2008). La democracia en América Latina: calidad y rendimiento. Revista Sistema, 203-204, 125-147.

Arseneaux, C. L. (1999). The military in Latin America: Defining the road ahead. En J. Buxton y N. Phillips (eds.), Developments in Latin American political economy. States, markets and actors (pp. 93-111). Manchester/New York: Manchester University Press.

Barreda, M. (2009). La calidad de la democracia en América Latina: Medición y claves explicativas. En Actas del IX Congreso de AECPA 2009. Recuperado de http://www.aecpa.es/uploads/ files/congresos/congreso_09/grupos-trabajo/ area04/GT05/02.pdf

Bayley, D. (1985). Patterns of policing. A comparative internacional analysis. New Brunswick: Rutgers University Press.
Bonilla, M. E. (2015). Democracia, desempeño institucional y cuerpos policiales. Revista Reflexión politica, 17 (33), 108-120. Recuperado de http:// www.redalyc.org/pdf/110/11040046009.pdf

Bonilla, M. E. (2016). Desempeño policial democrático: reto de la seguridad pública en América Latina. Revista Politica, globalidad y ciudadanía. Recuperado de http://revpoliticas.uanl.mx/RPGyC/ index.php/RPGyC/article/view/15/14

Cano, I. (2003). La policía y su evaluación. Propuestas para la construcción de indicadores de evaluación en el trabajo policial. Montevideo: Centro de Estudios para el Desarrollo. Área de Seguridad Ciudadana.

Cruz, J. (2010). Mala conducta policial y democracia en América Latina. Perspectivas desde el Barómetro de las Américas, 33. Recuperado de http://www. vanderbilt .edu/lapop/insights/I0833es.pdf

Collier, D. y Levitski, S. (1997). Democracy with adjectives: Conceptual innovations in comparative research. World Politics, 49 (3), 430-451.

Dahl, R. (1971). Poliarchy: Participation and Opposition. New Haven: Yale University Press.

Dahl, R. (2002). La democracia y sus críticos. Madrid: Paidós, Estado y Sociedad.

Dahl, R. (2007). La poliarquía. En A. Battle (coord.). Diez textos básicos de ciencia politica (pp. 77-92). Barcelona: Ariel.

Dammert, L. (2005). Reforma policial y participación militar en el combate a la delincuencia. Análisis y desafíos para América Latina. Revista Fuerzas Armadas y Sociedad, (1), 133-152. Recuperado de http://www.fasoc.cl/files/articulo/ ART43622189c08b8.pdf

Diamond, L. (1997). Is the third wave of democratization over? Assessment. Recuperado de http:// kellogg.nd.edu/publications/workingpapers/ wPs/236.pdf 
Diamond, L., Linz, J. y Lipset, S. (1990). Politics in Developing Countries. Lynne Rienner Publishers.

Frühling, H. (2001). La reforma policial y el procesos de democratización en América Latina. Montevideo: Centro de Estudios para el Desarrollo.

Frühling, H. (2003) Policía comunitaria y reforma policial en América Latina. ¿Cuál es el impacto? Centro de Estudios en Seguridad y Convivencia (CESC). Recuperado de http://www.cesc.uchile. cl/publicaciones/op_01_policiacomunitaria.pdf

Gallardo, M. (2006). Ética y deontología policial, policiología y metapoliciología. Madrid: Universidad Complutense de Madrid.

González, E. (2006). Sobre el concepto de represión. Revista de Historia contemporánea, (6). Recuperado de http://hispanianova.rediris.es/6/ dossier/6d022.pdf

Hales, G y Higgins, A. (2016). Priorisation in a changing world: seven challenges for policing. The Police Foundation. Recuperado de http://www.policefoundation.org.uk/uploads/holding/projects/ police_prioritisation.pdf

Herreda, A. (2006). Deontología policial: reflexiones y retos para las policías de la región americana. Santiago: Policía de Investigaciones de Chile Santiago.

Hough, M., Jackson, J., Bradford, B., Myhill, A. y Quinton, P. (2010). Procedural Justice, Trust and Institutional Legitimacy. Policing: A Journal of Policy and Practice, 4/3: 203-210.

Jackson, J., Bradford, B., Stanko, E. A. y Hohl, K. (2012). Just Authority? Trust in the Police in England and Wales. London: Routledge

Jar, G. (1999). El papel de la policía en una sociedad democrática. Revista Española de Investigaciones Sociológicas (REIS), 85.
Linz, J. y Stepan, A. (1996). Problems of democratic transition and consolidation: Souther Europe, South America, and post-communist Europe. Baltimore: Johns Hopkins University Press.

López, B. y Tudela, P. (2005). Marco conceptual para el estudio de politicas públicas de seguridad ciudadana. Los casos de Argentina, Chile y Uruguay. Washington: Banco Interamericano de Desarrollo.

López, E. y Frühling, H. (2008) Responsabilidad policial en democracia. Una propuesta para América Latina. México: Cambio xxI.

Mohor, A. (2007). Uso de indicadores para evaluar el funcionamiento policial. Santiago: Centro de Estudios en Seguridad Ciudadana. Universidad de Chile. Recuperado de https://www.cesc.uchile.cl/publicaciones/op_07_documentodos.pdf

Morlino, L. (2007). Explicar la calidad democrática: ¿qué tan relevantes son las tradiciones autoritarias? Revista de Ciencia politica, 27 (2), 3-22.

Morlino, L. (2009). Democracias y democratizaciones. Madrid: cIs.

Oficina de las Naciones Unidas Contra la Droga y el Delito (UNODC) (2010). Policía. Seguridad pública y prestación de servicios policiales. Recuperado de https://www.unodc.org/documents/justiceand-prison-reform/crimeprevention/Public_Safety_and_Police_Service_Delivery_Spanish.pdf Programa de las Naciones Unidas para el Desarrollo (PNUD) (2013). Informe Regional Desarrollo Humano 2013-2014. Seguridad ciudadana con rostro humano: diagnóstico y propuestas para América Latina. Washington: PNUD.

Reiner, R. (1998). Process or product? Problems of assessing individual police performance. En J. P. Brodeur (ed.), How to Recognize Good Policing. Sage \& Police Executive Forum (pp. 55-72). London: Sage Publications.

OPERA, $\mathrm{N}^{\circ}$ 22. Enero-Junio 2018.pp. 121-139 
Requena, I. (2016). La legitimidad policial y la colaboración ciudadana con la policía. Revista para el análisis del derecho. Recuperado de http://www. indret.com/pdf/1216.pdf

Rico, J. M. (1983). Policía y sociedad democrática. Madrid: Alianza Universidad.

Rueschemeyer, D., Di Huber, E. y Stephens, J. (1992). Capitalist development y democracy. Cambridge: Polity Press.

Saín, M. F. (2003). Seguridad, democracia y reforma de la organización policial en la Argentina. Condiciones institucionales, problemática delictiva y dilemas políticos. Buenos Aires: Fondo de Cultura Económica. Recuperado de http://xa.yimg.com/kq/ groups/21897551/86964068/name/Seguridad $+y+$ democracia+Sa\%C3\%ADn+(1).pdf

Schedler, A. (1998). What is democratic consolidation? Journal of Democracy, 9 (2), 91-107.

Seligson, M. A. y Smith, A. E. (eds.) (2010). Political culture of democracy. Democratic consolidation in the Americas in hard times: Report on the Americas. Nashville: LAPOP, Vanderbilt University.
Tudela, P. (2007). Sociedad y policía: desarrollo y retos de la función policial en las democracias latinoamericanas. Recuperado de http://www. policiadeinvestigacionesdechile.cl/cidepol/biblioteca/sociedadypolicia.pdf

Tudela, P. (2011). La medición del desempeño policial en Chile: consideraciones sobre la calidad de los servicios y el trato a víctimas y denunciantes. Conceptos, (22), 1-33. Recuperado de http://148.202.18.157/sitios/publicacionesite/ pperiod/republicana/pdf/ActaRep01/articulos39.pdf

Tudela, P. (2012). La evaluación del desempeño de la policía: explorando relaciones entre opinión pública y labor policial en Chile. Revista Criminalidad, 54 (1), 341-366.

United State Agency for International Development (USAID) (2014). The political culture of democracy in the Americas, 2014: Democratic governance across 10 years of Americas Barometer. Recuperado de https://www.vanderbilt. edu/lapop/ab2014/AB2014_Comparative_Report_English_V3_Updated_040517_W.pdf 